\title{
APPLICATION OF THE SYSTEMIC ANALYSIS FOR SOLVING THE PROBLEMS OF DEPENDENT EVENTS IN AGRICULTURAL LANDS - CHARACTERISTICS AND METHODOLOGY
}

\author{
V. Velkovski* \\ PhD student in Economic Academy "Dimitar A. Tsenov" - Svishtov, Bulgaria
}

\begin{abstract}
Systemic analysis for the first time provides a generalized problem-solving methodology based on the system concept.

By its nature, the problem-solving function is close to the highest levels of functional representation of human activity.

Therefore, this function and its serving methodology are most integrative and include all the private functions required for its implementation.

Systemic analysis, such as problem-solving methodology, claims to play the role of unifying all the necessary methods, knowledge and action to solve problems.

This is what determines his attitude to such areas as operations research, theory of statistical decisions, decision theory, organization theory, and others.

For the implementation of management functions, the methods of the search and discovery theory, statistical methods, factor analysis, experimental theories, methods of study of the operations, methods of forecasting and others can be used.

The assessment of the ratio of system analysis and its related disciplines is based on the contribution of the individual discipline in the methodology for solving the problem.

The main content of the system analysis is not in the formal mathematical apparatus describing the systems and the problem solving and it is not in the special mathematical methods but in the conceptual apparatus, its ideas, approach and formulations.

Systemic analysis defines the base nomenclature of the functions that need to be implemented to solve the problem, that is to say, it defines the structure of the process performed by the organization as opposed to the traditional approach to the organization that determines its administrative structure.

A / Types of problems

A problem is called the situation, which is characterized by the difference between the required (desired) output and the existing outcome.

Exit is necessary if it is absent threatens the existence and development of the system.

Existing output is provided by the existing system.

The desired output is achieved by the desired system. The problem may be to prevent a reduction in output or increase. The condition of the problem is set by the existing system (the "known").

The requirement - this is the desired system. The solution to the problem is what fills the difference between the existing and the desired system.

The system that fills this difference is the object of construction and is called a solution to the problem.

Problems can be manifested by symptoms. The systematic manifestation of symptoms is a tendency.

Detecting the problem is a result of identifying the symptoms.

Identification is possible provided that the desired behavior of the system is known. Once the problem has been identified, it is necessary to predict its development and to assess the timeliness of the decision, that is to say, what will be the state of the system for an unresolved problem.
\end{abstract}

Key words: Systemic analysis, management functions, Issue, Identification, operations research, model, inbound and outbound data, main purposes, Exploration of operations and abstract system.

\section{INTRODUCTION}

Systemic analysis provides a generic problemsolving methodology based on the system concept.

\footnotetext{
*Correspondence to: VALERI VELKOVSKI, PhD

student in Economic Academy "Dimitar A. Tsenov"

- Svishtov, Bulgaria, e-mail: jurist57@abv.bg
}

By its nature, the problem-solving function is close to the highest levels of functional representation of human activity.

Therefore, this function and its serving methodology are most integrative and include all the private functions required for its implementation. 
Systemic analysis as a problem-solving methodology claims to be the unifying role of all the necessary methods, knowledge and action to solve problems.

This is what determines his attitude to such areas as operations research, theory of statistical decisions, decision theory, organization theory, and others.

For the fulfillment of the management functions, the methods of the search and discovery theory, statistical methods, factor analysis, experimental theories, methods of study of the operations, methods of forecasting and others can be used.

The assessment of the ratio of system analysis and its related disciplines is based on the contribution of the individual discipline in the methodology for solving the problem.

The subject of the report is the application of the system analysis in the preparation and implementation of spatial events in agricultural lands.

There are some specificities in the system analysis technology in terms of defining and solving specific problems.

The aim of the author is to justify the necessity and usefulness of system analysis in the management of processes in agribusiness, respectively in the development processes.

\section{Types of organizational problems in the context of system analysis.}

The main content of the system analysis is not in the formal mathematical apparatus describing the systems and the problem solving and it is not in the special mathematical methods but in the conceptual apparatus, its ideas, approach and formulations.

System analysis determines the base nomenclature of functions that need to be implemented to solve the problem, ie. it defines the structure of the process performed by the organization as opposed to the traditional approach to the organization that determines its administrative structure.

\section{A / Types of problems.}

The problem is called the situation, which is characterized by the difference between the required (desired) exit and the existing outcome [Panov, T, M. Panova, Theory of the Organization, p.144, ed. RIC, Sofia-2005].
VELKOVSKI V.

Exit is necessary if it is absent threatens the existence and development of the system.

Existing output is provided by the existing system.

The desired output is achieved by the desired system. The problem may be to prevent a reduction in output or increase. The condition of the problem is set by the existing system (the "known").

The requirement - this is the desired system. The solution to the problem is what fills the difference between the existing and the desired system.

The system that fills this difference is the object of construction and is called a solution to the problem.

Problems can be manifested by symptoms. The systematic manifestation of symptoms is a tendency. Detecting the problem is a result of identifying the symptoms.

Identification is possible provided that the desired behavior of the system is known. Once the problem has been identified, it is necessary to predict its development and to assess the timeliness of the decision, ie. what will be the state of the system for an unresolved problem.

Assessing the timeliness of the solution to the problem makes it possible to determine the necessity of its solution.

The decision-making process focuses on iteratively performed operations to identify the conditions, goals, and opportunities to solve the problem.

The result of this identification is the description of the possibilities, objectives and terms of the terms of the input, output, process, feedback, constraints, link properties, ie. in the terms of the structures and their elements.

Depending on the depth of their identification and the opportunity to explore, the problems are divided into three classes:

a/ well-structured or quantifiable problems in which material dependencies are so well understood that they can be expressed by numbers or symbols that are ultimately numerically valued;

b/ unstructured or qualitative problems containing a description of only the most important resources, signs and characteristics, 
quantitative relationships between which are completely unknown;

c/ poorly structured or mixed issues that contain both qualitative and quantitative elements, dominating the qualitative, littleknown and undefined aspects of the problems.

B / Solving problems by using different methods.

In order to solve well-structured quantified problems, the methods of operation research are widely used.

The approach to exploring operations is based on the construction of mathematical models and the use of linear and non-linear programming methods, mass-theory theory, game theory, and others to find the optimal strategy for managing targeted actions.

The words "well structured problems" do not mean that these models are light. Building a mathematical model that reflects the main features of the problem is often accompanied by significant difficulties.

The main difficulty in using the methods of investigating operations to solve military, economic, technical and other tasks is, in addition to properly selecting the model, to collect the necessary information to solve the tasks - incoming and outgoing data, conditions for solving, etc., in their analysis and assurance that the chosen model reflects the essence of the task.

Most unstructured problems are solved using heuristic methods, which lack some sort of logical procedure for finding a solution.

Between well-structured and unstructured problems is the class of poorly structured problems. Systematic analysis is designed to solve precisely such problems that are addressed by most of the most important economic, technical, political and military strategic tasks of a very large scale.

Typically, poorly structured problems include problems with the following features:

a / the solutions identified relate to the future;

$\mathrm{b} /$ the range of possible alternatives is too wide;

c/ decisions depend on the current incompleteness of technological advances;

$\mathrm{d} /$ the decisions taken require large investments and contain elements of risk; e / the value and time for solving the problem are not fully determined;

$\mathrm{f} /$ Internally, the problem is too complicated, due to the need to combine different resources used to solve it.

By applying the system analysis in the process of structuring the problem, some non-element elements, subtasks, receive a quantitative description and relations between all elements begin to become increasingly defined.

Based on this, unlike the use of the methods for investigating operations in the application of the system analysis, the initial purity and thorough formulation of the problem is not mandatory.

This clarity must be achieved in the process of analysis itself and is seen as one of its main objectives.

Operations research can be used to solve individual subtasks occurring at moderate and lower levels of governance, covering a relatively short period of time, primarily related to the use and allocation of the organization's internal resources.

Such tasks contain a large number of controllable and a small number of uncontrollable variables, and in many cases can be presented quantitatively and solved with a computer.

On the contrary, strategic issues related to the development of long-term production policy, new industries, the allocation of common resources, etc. can not be formalized as a taskresearch task.

Problems of this kind are subject to system analysis. They are of a long-term nature, they encompass the organization all the way and influence its situation in the future, too many factors are external to this organization.

Systemic analysis contains elements inherent in both rigorous co-decision methods and an intuitively heuristic approach, entirely dependent on the researcher.

The change that is taking place today is that art changes its form, transforming itself from an approach based on accidental inspiration and intuition, into an approach based on the analysis that is supported by intuition and experience. 
The distinctive feature of the new approach is the individual clearing of decision-making methods and the use of these methods at high political levels.

\section{Essence of system analysis.}

The notion of systemic is used because the study of weakly structured problems itself is based on the category of system under which the system analysis methodology is understood to be the set of elements organized for the implementation of a number of prescribed functions in order to achieve the desired results [Panov, T, M. Panova, Theory of the Organization, p148, ed. RIC, Sofia-2005].

On the one hand, the system is the physical reality that needs to be decided.

On the other hand, in the process of system analysis, an abstract or conceptual system is described, described by means of symbols or other means, which is a structurally logical device, the purpose of which is to serve as a tool for understanding, description and possible optimization of of the real physical system-organization.

Such an abstract system can be a mathematical, machine or verbal model, or a system of models, a human machine game, a program such as a description of a set of purposeful functions and actions, and so on.

In the physical and the corresponding abstract system there must be a mutually unambiguous correspondence between the elements and their relationships.

In this case, it is possible and without the help of experts in real physical systems to assess the different working hypotheses regarding the appropriateness of one or other actions and using the corresponding abstract system, to make the most preferred solutions.

The term analysis is characterized by the actual investigation procedure, which consists of: breaking the whole problem of its constituent parts, which are more accessible to solving; the use of the most appropriate special methods for solving individual problems; the unification of private solutions, which is the general solution to the problem.

Obviously, in the process of system analysis, methods of analysis and synthesis intertwine with each other. During the analytical procedure, attention is always paid to the ways of combining the individual results into one
VELKOVSKI V.

and the influence of each element on the other elements of the system.

The main logical elements of this method in the process of system research are:

a / the purpose or set of objectives;

$\mathrm{b} /$ the alternative means by which the target can be reached;

c / the resources required for the creation and use of each system;

$\mathrm{d} /$ the mathematical and logical model or models, that is to say, the system of links between objectives, alternative means of achieving them, the environment and resources;

e / the criteria for choosing the preferred alternative. The criterion usually establishes a relationship between the goals and costs of achieving them.

Strictly speaking, the listed elements should not be considered a specific tool of system analysis. They are the basis of any analytical decision-making process, incl. and of the methodology for the analysis of operations and analysis.

In the study of the operations, the main focus is on the mathematical methods of finding the best strategies, assuming that the elements of the problem solved are sufficiently quantified from the very beginning of the study or that in the process of the operational research the outgoing data will be corrected and supplemented by analyzing the results of the decisions.

The approach to analysis is mainly constrained by the need to separate goals, alternatives, costs and criteria, but does not seek to establish the link between successful actions, methods of analysis and concepts of achieving them, indicating only what should be done is done without saying how to do that.

At best, his instrument is the empirical decision-making rules based on past experience.

Systemic analysis is a methodology for analyzing and solving problems using systemic research and comparison of alternatives that are performed on the basis of the cost-to-cost ratio for their implementation and the expected results.

Although logical and mathematical methods are often used in system analysis to solve problems, it is not to be supposed that there is 
a binding relationship between system analysis and complex mathematical apparatus.

Systematic analysis, unlike other more or less formalized approaches to substantiating management decisions, implies:

a / systematic study and mutual comparison of the alternative actions that lead to the achievement of the desired objectives;

$\mathrm{b} /$ comparing alternatives on the basis of the value of the resources spent and the benefit obtained from each alternative;

c / reporting and detailed analysis of uncertainties.

\section{CONCLUSION}

The management of agribusiness as a whole and in particular of land-use planning activities need active application of system analysis, especially since the management cycle in agribusiness is seen by some authors as a system of interconnected functions in relation to the taking and implementation of management solutions [Nikolova M., M. Linkova, V. Blajeva, R. Nenova, Agrarian Management, p.12, Acad. Tsenov, Svishtov, 2018).

By applying the system analysis in the management of organizational events, a number of problems, disturbances and uncertainties can be solved and eliminated also in the environment for entrepreneurship [Nikolova M., M. Linkova, R. Nenova, Agrarian Entrepreneurship, p.34, Academic publishing, Tsenov, Svishtov, 2017].

As a problem-solving methodology, system analysis indicates the essentially necessary sequence of interdependent operations, which is expressed in the following: revealing the
VELKOVSKI V.

problem, constructing the solution to the problem, and realizing this solution.

In more detail, the private functions associated with the solution of the problem are the following: identification of symptoms, determination of the actuality of the problem, determination of the purpose, revealing the structure of the system and its defective elements, defining the structure of the possibilities, finding alternatives, evaluation of alternatives, selection of alternatives, making decisions, recognition of the decision by the team, managers and contractors, realization of the decision, evaluation of the realization and its consequences.

This can be expressed in more detail through the following stages of the problem solving process:
a / formulating the problem:
b / collecting data describing the problem;
$\mathrm{c} /$ solving the problem.

Practice has shown that the duration of the first two phases may exceed the duration of the third phase by approximately one hundred percent.

\section{REFERENCES}

1. Nikolova M., M. Linkova, V. Blajeva, R. Nenova, Agricultural Management, p.12, Academic publishing, Tsenov, Svishtov, 2018;

2. Nikolova M., M. Linkova, R. Nenova, Agrarian Entrepreneurship, p.34, Academic publishing, Tsenov, Svishtov, 2017;

3. Panov, T, M. Panova, Theory of the Organization, p148, ed. RIC, Sofia-2005. 